Sanofi Aventis, Inventiva, CSL Behring, Boehringer Ingelheim, Bayer, Robert Lafyatis: None declared, David Fox: None declared, Dinesh Khanna Shareholder of: Eicos Sciences, Inc, Grant/research support from: Bayer, BMS, Pfizer, Horizon, Consultant for: Actelion Acceleron, Arena, Bayer, BI, BMS, CSL Behring, Corbus, Cytori, GSK, Genentech/Roche, Galapagos, Employee of: Elcos Sciences, Inc, Michael Whitfield Shareholder of: Scientific Founder, Celdara Medical LLC, Grant/research support from: Dr. Whitfield has received research contracts from Celdara Medical LLC and UCB Biopharma, Consultant for: Bristol Myers Squib, Celdara Medical, Corbus, and Boerhinger Ingelheim

DOI: 10.1136/annrheumdis-2019-eular.7661

\section{THU0355 IGURATIMOD MIGHT TREAT SCLERODERMA WITH INTERRUPTED EGR1/TGF-B LOOP}

Qingran Yan ${ }^{1}$, Lichong Shen ${ }^{1}$ Daniel Morales-Cano ${ }^{1}$ Xiaoxiang Chen ${ }^{1} .{ }^{1}$ Renji Hospital, Shanghai Jiaotong University, School of Medicine, Department of Rheumatology, Shanghai, China

Background: Systemic sclerosis (SSc) is an autoimmune disease characterized with multiple organ fibrosis. Previous studies showed transcription factor early growth response 1 (Egr1) overexpressed in the lesional skin of SSc patients, as well as Egr1 inducible genes ${ }^{1}$. Egr1 forms a positive feedback loop with master pro-fibrotic cytokine TGF $\beta$ and thus promotes fibrosis.

Objectives: To investigate the anti-fibrotic effect of a novel DMARD iguratimod in scleroderma models and patient skin grafts.

Methods: We used iguratimod to treat TGF $\beta$-stimulated human skin fibroblast, bleomycin induced mice, tight skin 1 (TSK-1) mice and SSc skin grafts. The bleomycin model contained pre-establish fibrosis and late onset treatment. The skin grafts came from three SSc patients and was planted into irradiated nude mice. Results: Iguratimod down-regulated egr1 expression in human skin fibroblast, with decreased collagen production and $\alpha$-SMA expression. Knocking down Egr1 in fibroblast could mimic these effects. Both oral and topical iguratimod could reduce dermal thickening and collagen deposition in bleomycin induced skin fibrosis. $\alpha$-SMA (+) myofibroblast counts, as well as Egr1 (+) and/or TGF $\beta$ (+) fibroblast counts in iguratimod treated groups were significantly less than the controls. Similarly, topical iguratimod ameliorated fibrosis with deduced dermal thickening in TSK-1 mice. Of note, 5-week iguratimod local injection remarkably reduced collagen content in skin grafts from three SSc patients. Staining of Egr1 and TGF $\beta$ in skin tissue were inhibited after iguratimod treatment simultaneously.

Conclusion: We found the potential of iguratimod to treat SSc, which was characterized as an Egr1 inhibitor. Further clinical investigation is needed to establish its safety and efficacy.

\section{REFERENCES}

[1] Bhattacharyya S, Sargent JL, Du P, et al. Egr-1 induces a profibrotic injury/repair gene program associated with systemic sclerosis. PloS one 2011;6(9):e23082 doi: 10.1371/journal.pone.0023082

[2] Chen SJ, Ning H, Ishida W, et al. The early-immediate gene EGR-1 is induced by transforming growth factor-beta and mediates stimulation of collagen gene expression. The Journal of biological chemistry 2006;281 (30):21183-97 doi: 10.1074/jbc.M603270200

[2] Wu M, Melichian DS, de la Garza M, et al. Essential roles for early growth response transcription factor Egr-1 in tissue fibrosis and wound healing. The American journal of pathology 2009;175(3):1041-55 doi: 10.2353/ ajpath.2009.

Acknowledgement: This work is supported by National Natural Science Foundation of China (81601401).

Disclosure of Interests: None declared

DOI: 10.1136/annrheumdis-2019-eular.2421

\section{THU0356 STAPHYLOCOCCUS AUREUS REGULATES FIBROBLAST FUNCTIONS - IMPLICATIONS FOR TISSUE REPAIR IN CHRONIC DIGITAL ULCERS IN SYSTEMIC SCLEROSIS}

Masaya Yokota ${ }^{1}$, Matthew Kassier ${ }^{1}$, Nicola Häffner ${ }^{2}$, Matthias Brunner ${ }^{1}$, Janine Schniering ${ }^{1}$, Oliver Distler ${ }^{1}$, Annelies S. Zinkernagel ${ }^{2}$, Britta Maurer ${ }^{1}$. ${ }^{1}$ Center of Experimental Rheumatology, Department of Rheumatology, University Hospital Zurich, Zurich, Switzerland; ${ }^{2}$ Department of Infectious Diseases and Hospital Epidemiology, University Hospital Zurich, University of Zurich, Zurich, Switzerland

Background: Chronic digital ulcers (DU) are a major complication in systemic sclerosis (SSc). Non-healing wounds are characterized by persistent inflammation, defective re-epithelialization and impaired matrix remodeling, and are often accompanied by bacterial colonization. In DU, the breach of the basement membrane exposes tissue and cell layers to commensa skin bacteria.

Objectives: To investigate whether interactions between commensal skin bacteria and dermal fibroblasts affect tissue repair mechanisms.

Methods: Dermal fibroblasts isolated from healthy controls $(\mathrm{HC})$ and patients with diffuse cutaneous SSc (dcSSc) ( $n=3$, each) were co-cultured with Staphylococcus aureus (SA) for $3 \mathrm{~h}$ at using a lower inoculum rather representing colonization $\left(1 \times 10^{5} \mathrm{CFU} / \mathrm{ml}\right)$ and a higher inoculum reflecting infection $\left(1 \times 10^{7} \mathrm{CFU} / \mathrm{ml}\right)$. Thereafter, fibroblasts were cultured with flucloxacillin-containing medium to kill extracellular and adherent bacteria. For mechanistic studies, a fibroblast cell line (BJ-5ta) was used. IL 6 , IL-8, pro-collagen $\mid \alpha$, and interferon (IFN)- $\beta$ proteins in culture supernatants were measured by ELISA. Gene expression was assessed by quantitative PCR. Contractility was analyzed by collagen gel contraction assay. Wound closure assay was performed on a confluent cell layer with a uniform cell-free gap, generated by a cell culture insert. Expression of alpha-smooth muscle actin ( $\alpha$-SMA) was evaluated by Western blotting, cell proliferation with a colorimetric assay. Cell death was analyzed by live cell real time assay. Bacterial invasion was assessed by immunofluorescence staining using confocal microscopy and CFU recovery. Gene knockdown was performed using siRNA.

Results: Exposure to $1 \times 10^{7} \mathrm{CFU} / \mathrm{ml}$ of $\mathrm{SA}$ influenced fibroblast function. In brief, exposure to SA increased the secretion of IL-6 and IL-8 in derma fibroblasts by $1571 / 455$-fold (HC/dcSSc; $p=0.08 /<0.05$ ) and by $325 / 1402$-fold ( $\mathrm{HC} / \mathrm{dcSSc}$; $\mathrm{p}<0.05$ each) respectively. In addition we observed upregulation of the expression of MMP1 by $8.2 / 5.7$-fold (HC/dcSSc; $p<0.05$ each) and MMP3 by $5.1 / 5.7$-fold $(\mathrm{HC} / \mathrm{dcSS}$; $\mathrm{p}=0.06 /<0.05)$. There was a trend towards decreased secretion of pro-collagen $1 \alpha$. TGF- $\beta$-induced $\alpha$-SMA expression was completely inhibited. Cell contraction was impaired by $22.0 / 31.6 \%$ (HC/dcSSc $\mathrm{p}=0.08 /<0.05$ ), wound closure by $18.8 / 14.9 \%$ (HC/dcSSc; $\mathrm{p}<0.05$ each), and proliferation by $30.5 / 44.3 \%$ (HC/dcSSc; $p=0.24 /<0.05)$. SA induced apoptosis and necrosis. SA invaded fibroblasts via endocytosis $(25-50 \%$ of inoculated live SA). IFN- $\beta$ secretion was induced 8.4/31.7-fold (HC/dcSSc; $p<0.05$ each). The genes of cytosolic dsDNA sensor molecules such as cyclic GMP-AMP synthase (cGAS) were upregulated 11.9/8.6-fold (HC/dcSSc; $\mathrm{p}<0.05$ each). TLR9, an endosomal DNA sensor, was constitutively expressed. Knockdown of STING or MyD88, downstream mediators of cGAS and TLR9 respectively, reduced the induction of IFN- $\beta$ (down to 68.2/66.2\%; $\mathrm{p}=0.20 / 0.16$ ), IL-6 (down to $57.2 / 55.2 \% ; \mathrm{p}<0.05$ each), and IL-8 (down to $45.3 / 36.0 \% ; \mathrm{p}<0.05$ each). Furthermore, inhibition of endocytosis with cytochalasin $D$ decreased the expression of IFN- $\beta$ (down to $24.6 \%$; $<<0.05$ ), IL-6 (down to $34.9 \%$; $p<0.05$ ), and IL-8 (down to 27.6\%; $\mathrm{p}<0.05$ ). In contrast, inhibition of endocytosis did not affect induction of apoptosis. This suggests that the invasion of fibroblasts by $\mathrm{SA}$ and the subsequent intracellular DNA sensing are crucial for the induction of these genes independently of apoptosis.

Conclusion: Invasion of dermal fibroblasts by SA with activation of the STING and TLR9-MyD88 pathways is a key element in the impairment of tissue repair responses.

\section{THU0357 VENTRICULAR-ARTERIAL COUPLING AS A PREDICTOR OF CARDIOVASCULAR EVENTS IN SYSTEMIC SCLEROSIS}

Elisabetta Zanatta ${ }^{1}$, Pamela Polito ${ }^{1}$, Giulia Famoso ${ }^{2}$, Franco Cozzi ${ }^{1}$, Andrea Doria ${ }^{1}$, Francesco Tona ${ }^{2} .{ }^{1}$ Rheumatology Unit, Department of Medicine DIMED, Padova, Italy; ${ }^{2}$ Department of Cardiac, Thoracic and Vascular Sciences, University of Padova, Padova, Italy

Background: Standard Transthoracic Echocardiography (TTE) parameters have shown a low sensitivity in the detection of primary miocardia involvement (PMI) in systemic sclerosis (SSc). Arterial-ventricular coupling (VAC) is calculated by TTE as the ratio between arterial elastance (Ea) and ventricular end-systolic elastance (Ees), which reflects left ventricle stifness. VAC is a central determinant of cardiovascular performance.

Objectives: We aimed to assess TTE-derived measures of cardiac mechanics and the prognostic role of VAC in SSc.

Methods: 75 patients affected by SSc without symptoms of cardiac involvement and 10 controls matched for sex and age were retrospectively evaluated. We considered the first TTE available after SSc diagnosis. Determinants of cardiac mechanics were calculated by TTE ${ }^{1}$ : end systolic elastance (Ees), arterial elastance (Ea), ventricular arterial cou pling $(\mathrm{VAC}=\mathrm{Ees} / \mathrm{Ea})$, end diastolic elastance $(\mathrm{Eed})$. A value of VAC > 0.62 was considered indicative of altered VAC (i.e. uncoupling), whereas a lower value as normal (i.e. coupling). Finally, we considered the hospitalization for cardiovascular event during a mean follow-up of $10.8 \pm 1.8$ years. 
Results: Among the 75 patients 42 (59.2\%) had a diffuse cutaneous form of SSc (dSSc) and $64(85.3 \%)$ were female; in SSc patients the mean age was $53 \pm 12.1$ yrs and the mean disease duration $10.1 \pm 2.3$ yrs. $36(52.2 \%)$ patients had positive SCI70, 31 (44.9\%) ACA, and 2 (2.9\%) anti-RNA pol III. Compared to controls, SSc patients had higher Ea (2.28 vs $0.95 \mathrm{mmHg} / \mathrm{ml}, \mathrm{p}=0.003$ ) and Ees $(3.95$ vs $2.98 \mathrm{mmHg} / \mathrm{ml}$, $\mathrm{p}=0.05)$, and increased diastolic stiffness (Eed) $(0.210$ vs $0.146 \mathrm{mmHg} / \mathrm{ml}$, $\mathrm{p}=0.01$ ). VAC was consequently comparable to controls. SSc patients affected with dcSSc had a lower Ees (2.90 vs 4.367, $\mathrm{p}<0.001)$ and Eed (0.24 vs $0.17, p=0.032$ ) and a higher VAC (0.52 vs $0.70, p=0.01$ ) compared to ISSc. No differences were found between patients with antiScl70 and ACA. At 10 years, $23 \%$ of patients was hospitalized for at least one cardiovascular event. The analysis of survival free hospitalization or death in all SSc patients demonstrated a worse outcome and poor prognosis in patients with an altered VAC (31, 47.7\%) compared to those with a normal VAC (34, 52.3\%) (Figure 1)

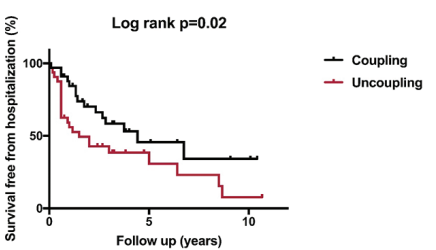

Abstract THU0357 - Figure 1

Conclusion: Our study suggests that both ventricular and arterial stifness may be increased in SSc patients without signs and symptoms of heart disease. Since VAC seems to have a prognostic role in the prediction of cardiovascular events in SSc, it could be helpful to define an early therapeutic strategy to prevent or delay cardiac manifestations in these patients.

\section{REFERENCE:}

1. Sagawa K et al, Am J Cardiol 1977;40:748-753.

Disclosure of Interests: None declared

DOI: 10.1136/annrheumdis-2019-eular.7479

\section{Spondyloarthritis - clinical aspects (other than treatment)}

\section{THU0358 DEVELOPMENT OF A SET OF ASAS QUALITY STANDARDS FOR ADULTS WITH AXIAL SPONDYLOARTHRITIS}

Uta Kiltz $^{1,2}$, Robert B.M. Landewé ${ }^{3}$, Désirée van der Heijde ${ }^{4}$, Martin Rudwaleit ${ }^{5}$, Maxime Dougados ${ }^{6}$, Nurullah Akkoc ${ }^{7}$, Annelies Boonen ${ }^{8}$, Jan Brandt-Juergens ${ }^{9}$ Philippe Caroon ${ }^{10}$, Laure Gossec ${ }^{11}$, Merryn Jongkees ${ }^{12}$, Pedro Machado ${ }^{13}$, Helena Marzo-Ortega ${ }^{14}$, Anna Moltó ${ }^{6}$, Victoria Navarro-Compán ${ }^{15}$, Karin Niedermann Schneider ${ }^{16}$, Percival D. Sampaio-Barros ${ }^{17}$, Gleb Slobodin ${ }^{18}$, Filip van den Bosch ${ }^{19}$, Astrid van Tubergen ${ }^{8}$, Salima van Weely ${ }^{20}$, Michael Weisman ${ }^{21}$, Dieter Wiek ${ }^{22}$, Juergen Braun ${ }^{1,2}$. ${ }^{1}$ Rheumazentrum Ruhrgebiet, Herne, Germany; ${ }^{2}$ Ruhr-University, Bochum, Germany; ${ }^{3}$ ARC, Amsterdam, Netherlands; ${ }^{4}$ Leiden University Medical Center, Leiden, Netherlands; ${ }^{5}$ Klinikum Bielefeld, Bielefeld, Germany; ${ }^{6}$ Hôpital Cochin, Paris, France; ${ }^{7}$ Rheumatology, Izmir, Turkey, ${ }^{8}$ Maastricht University Medical Center, Maastricht, Netherlands; ${ }^{9}$ Rheumapraxis, Berlin, Germany, ${ }^{10}$ Ghent University, Gent, Belgium; ${ }^{11}$ Sorbonne Universite, Hopital Pitie-Salpetriere, Paris, France; ${ }^{12}$ Seayn Medical, The Hauge, Netherlands; ${ }^{13}$ University College London, London, United Kingdom; ${ }^{14}$ Leeds General Infirmary, Leeds, United Kingdom; ${ }^{15}$ University Hospital La Paz, IdiPaz, Madrid, Spain;

${ }^{16}$ ZHAW Zurich University, Zurich, Switzerland; ${ }^{17}$ University Sao Paulo, Sao Paulo, Brazil; ${ }^{18}$ Bnai-Zion Medical Center, Haifa, Israel; ${ }^{19}$ Ghent University Hospital, Gent, Belgium; ${ }^{20}$ Leiden University Medical Centre, Leiden, Netherlands; ${ }^{21}$ Cedars-Sinai Medical Center, Los Angeles, United States of America; ${ }^{22}$ Rheuma-Liga, Cologne, Germany

Background: There is wide variation in the management of patients with axial spondyloarthritis (axSpA) worldwide with significant unmet needs such as delayed diagnosis. A major goal of the international organization Assessment of SpondyloArthritis international Society (ASAS) is to improve quality of care and health outcomes in axSpA. One way to achieve this is to define quality standards (QS) in order to identify resources and processes which may need to be optimized. Such standards must be specific, measurable, aspirational and achievable in daily care.

Objectives: To develop ASAS QS to ultimately improve the quality of care for adults with axSpA.

Methods: The ASAS QS group, established in 2015, developed a stepwise approach starting with (I) an overview and open discussions resulting in a proposal for possible key areas for quality improvement. Thereafter, (II) ASAS members and invited patients discussed and commented on a provisional list via a web-based survey with the possibility to propose additional key areas for quality improvement. (III) The complete list was then evaluated by ASAS members and invited patients. (IV) Then, the ASAS QS group prioritized key areas for which quality statements and measures are to be developed, and (V) phrased QS for the most important key areas. Finally (VI), a draft version was commented on, discussed and finally agreed by the ASAS members at the Annual ASAS Meeting 2019.

Results: The ASAS QS group, consisting of 20 rheumatologists, 2 physiotherapists and 2 patients, provided 34 potentially key areas for quality improvement which were commented by 140 participants (86 physicians, 42 patients). Within that process 3 new key areas were proposed and all 37 key areas for improvement were again evaluated by 120 participants (86 physicians, 29 patients). Five key areas were identified to be most important to phrase QS: referral, rheumatologic assessment, treatment, edu cation/self-management and comorbidities. Altogether, 9 QS, each accompanied by a rationale and a measure (figure), were endorsed by ASAS.

Conclusion: ASAS successfully developed the first QS set for improve ment of health care provided for adults with axSpA. All QS are achievable in daily care in an optimized situation and intend to minimize variation in quality of care. It is emphasized that ASAS is well aware that all QS are ideal visions of an optimal care which may currently not be realistic in many countries.

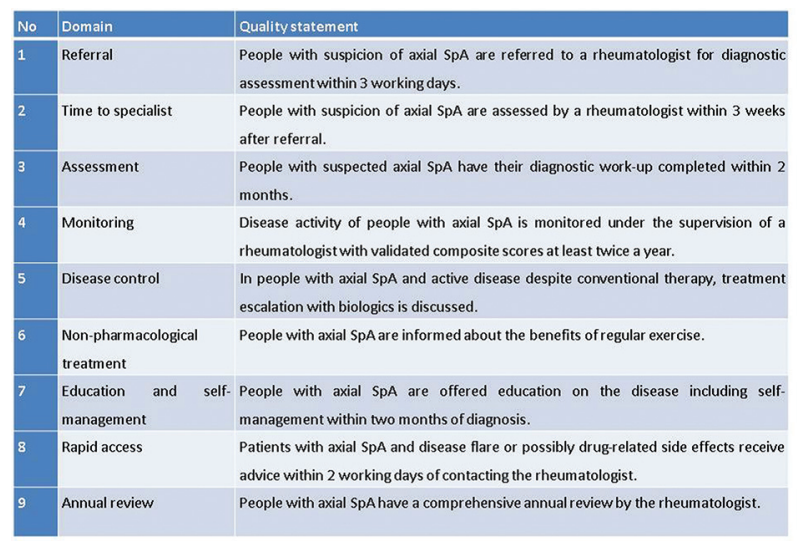

Abstract THU0358 - Figure 1. ASAS quality standards for patients with axial spondyloarthritis

Disclouser of Interest: Uta Kiltz Grant/research support from: AbbVie, Chugai, Eli Lilly, Grünenthal, Janssen, MSD, Novartis, Pfizer, Roche, and UCB. Consultant for: AbbVie, Chugai, Eli Lilly, Grünenthal, Janssen, MSD, Novartis, Pfizer, Roche, and UCB., Robert B.M. Landewé: None declared, Désirée van der Heijde Consultant for: AbbVie, Amgen, Astellas, AstraZeneca, Bristol-Myers Squibb, Boehringer Ingelheim, Celgene, Daiichi, Eli-Lilly, Galapagos, Gilead, GlaxoSmithKline, Janssen, Merck, Novartis, Pfizer, Regeneron, Roche, Sanofi, Takeda, Union Chimique Belge, Martin Rudwaleit Consultant for: AbbVie, BMS, Celgene, Janssen, Eli Lilly, MSD, Novartis, Pfizer, Roche, UCB Pharma, Consultant for: AbbVie, BMS, Celgene, Janssen, Eli Lilly, MSD, Novartis, Pfizer, Roche, UCB Pharma, maxime dougados Grant research support from: Eli Lilly and Company, Pfizer, AbbVie, and UCB Pharma, Consultant for: Eli Lilly and Company, Pfizer, AbbVie, and UCB Pharma, Nurullah Akkoc: None declared, Annelies Boonen: None declared, Jan Brandt-Juergens: None declared, Philippe Caroon: None declared, Laure Gossec Grant/research support from: AbbVie, BMS, Celgene, Janssen, Lilly, MSD, Novartis-Sandoz, Pfizer, Sanofi, and UCB, Consultant for: AbbVie, Biogen, BMS, Celgene, Janssen, Lilly, MSD, Nordic Pharma, Novartis-Sandoz, Pfizer, Roche, Sanofi, and UCB, Consultant for: L Gossec has received honoraria from Celgene as investigator for this study, Merryn Jongkees: None declared, Pedro Machado Consultant for: Abbvie, BMS, Celgene, Janssen, MSD, Novartis, Pfizer, Roche and UCB, Speakers bureau: Abbvie BMS, Celgene, Janssen, MSD, Novartis, Pfizer, Roche and UCB, Helena Marzo-Ortega Grant/research support from: Janssen, Novartis and Pfizer Consultant for: AbbVie, Celgene, Janssen, Eli-Lilly, Novartis and UCB 